\title{
Multiattribute Group Decision Making with Unknown Decision Expert Weights Information in the Framework of Interval Intuitionistic Trapezoidal Fuzzy Numbers
}

\author{
Zongcai Jiang' and Yan Wang ${ }^{2}$ \\ ${ }^{1}$ Department of Mathematics and Information Science, Henan University of Economics and Law, Zhengzhou 450046, China \\ ${ }^{2}$ Department of Computer Science, Guangdong Polytechnic Normal University, Guangzhou 510665, China
}

Correspondence should be addressed to Zongcai Jiang; zongcaijiang@huel.edu.cn

Received 19 March 2014; Accepted 16 July 2014; Published 5 August 2014

Academic Editor: Tofigh Allahviranloo

Copyright (C) 2014 Z. Jiang and Y. Wang. This is an open access article distributed under the Creative Commons Attribution License, which permits unrestricted use, distribution, and reproduction in any medium, provided the original work is properly cited.

\begin{abstract}
The aim of this paper is to investigate an approach to multiattribute group decision making with interval intuitionistic trapezoidal fuzzy numbers, in which the decision expert weights are unknown. First, we introduce a distance measure between two interval intuitionistic trapezoidal fuzzy matrixes, and based on the distance between individual matrix and extreme matrix, as well as the average matrix, we obtain the decision expert weights. Second, we utilize the interval intuitionistic trapezoidal fuzzy weighted geometric (IITFWG) operator and the interval intuitionistic trapezoidal fuzzy ordered weighted geometric (IITFOWG) operator to aggregate all individual interval intuitionistic trapezoidal fuzzy decision matrices into a collective interval intuitionistic trapezoidal fuzzy decision matrix and then derive the group overall evaluation values of the given alternatives. Finally, an illustrative example of emergency alternatives selection is given to demonstrate the effectiveness and superiority of the proposed method.
\end{abstract}

\section{Introduction}

In 1986, Atanassov proposed the concepts of the intuitionistic fuzzy set (IFS), which is depicted by a membership function, a nonmembership function, and a hesitancy function $[1,2]$. IFS is more suitable for dealing with fuzziness inherent in decision data and decision information than Zadeh's fuzzy set [3]; therefore the investigation of multiattribute decision making with intuitionistic fuzzy information has received a great attention of researchers worldwide [4-11]. Some authors extended the IFS in another way, which extended a discrete set to a continuous one, such as triangular intuitionistic fuzzy number (TIFN) [12-15], intuitionistic trapezoidal fuzzy number (ITFN) [16, 17], and interval intuitionistic trapezoidal fuzzy number (IITFN) [18].

Wan and Dong [19] gave the definitions of ITFN about expectation and expectant score, hybrid aggregation operator, and ordered weighted aggregation operator. Ye [20, 21] presented an intuitionistic trapezoidal fuzzy multicriteria decision making method based on expected values and multicriteria group decision making method based on the similarity measures between ITFNs, respectively. Wan et al. [22] proposed a possibility mean-variance based method for intuitionistic trapezoidal fuzzy group decision making. Zhang et al. [23] investigated a grey relational projection method for multiattribute decision making based on intuitionistic trapezoidal fuzzy numbers.

Wan $[24,25]$ studied the IITFN in depth. He not only discussed the operational laws and properties of IITFN, giving a series of definitions of it, such as the score function, weighted arithmetic average operator, weighted geometric average operator, and the Hamming and Euclidean distances for interval-valued trapezoidal intuitionistic fuzzy numbers, but also established a multiattribute decision making model based on IITFN. Wei et al. [26] put forward some new aggregation operators of IITFN anddeveloped a multiple attribute group decision making approach with known decision expert weights information. 
In the existing literature, few studies have been carried out on IITFN. For the membership function value and nonmembership value of IITFN depending on different interval number, IITFN can better reflect decision maker's preference and express a different dimension of decision making information. Thus, it provides a new idea to solve the multiattribute decision making problems effectively.

To reflect the decision making more scientifically and democratically, in some large or important decision making problems, it often requires multiple decision makers to participate. In the process of group decision making, decision expert weights play an important role to aggregate all individual decisions into a collective decision because the decision makers may have different abilities, interests, hierarchical ranks, and so forth.

Due to the limited knowledge of the authors, no research has been carried out to handle multiattribute group decision making problems with unknown decision maker weights in the framework of IITFN. So this paper proposed a new group decision making methodology based on distance measure to derive the weights of experts, in which the attribute values take the form of interval intuitionistic trapezoidal fuzzy numbers.

To do that, the structure of this paper is organized as follows. A brief introduction of the IITFN theory is given in Section 2. In Section 3, we develop a new multiattribute group decision making method with incompletely known decision expert weights information under IITFN. In Section 4, we illustrate our proposed method with an example. Final conclusions are shown in Section 5.

\section{Preliminaries}

In the following, we will introduce some basic concepts related to interval intuitionistic trapezoidal fuzzy numbers.

Definition 1 (see [27]). Let $\widetilde{\beta}$ be an intuitionistic trapezoidal fuzzy number; its membership function is

$$
\mu_{\widetilde{\beta}}(x)= \begin{cases}\frac{x-a}{b-a} \mu_{\tilde{\beta}}, & a \leq x \leq b ; \\ \mu_{\widetilde{\beta}}, & b \leq x \leq c ; \\ \frac{d-x}{d-c} \mu_{\tilde{\beta}}, & c \leq x \leq d ; \\ 0, & \text { others. }\end{cases}
$$

Its nonmembership function is

$$
v_{\widetilde{\beta}}(x)= \begin{cases}\frac{b-x+v_{\tilde{\beta}}(x-a)}{b-a}, & a \leq x \leq b ; \\ \frac{v_{\tilde{\beta}},}{x-c+v_{\widetilde{\beta}}(d-x)} & b \leq x \leq c ; \\ \frac{d-c}{-c} & c \leq x \leq d ; \\ 0, & \text { others, }\end{cases}
$$

where $0 \leq \mu_{\tilde{\beta}} \leq 1,0 \leq v_{\tilde{\beta}} \leq 1,0 \leq \mu_{\tilde{\beta}}+v_{\tilde{\beta}} \leq 1$, and $a, b, c, d \in R$.

Then $\widetilde{\beta}=\left([a, b, c, d] ; \mu_{\tilde{\beta}}, v_{\tilde{\beta}}\right)$ is called an intuitionistic trapezoidal fuzzy number (ITFN).

If $\mu_{\widetilde{\beta}} \subset[0,1], v_{\widetilde{\beta}} \subset[0,1]$ are interval numbers and $0 \leq \sup \left(\mu_{\tilde{\beta}}(x)\right)+\sup \left(v_{\tilde{\beta}}(x)\right) \leq 1$, for convenience, let us denote $\mu_{\tilde{\beta}}(x)=[\mu, \bar{\mu}], v_{\widetilde{\beta}}(x)=[\underline{v}, \bar{v}]$. Then $\tilde{\beta}=$ $([a, b, c, d] ;[\mu, \bar{\mu}],[\underline{v}, \bar{v}])$ is called an interval intuitionistic trapezoidal fuzzy number (IITFN).

Definition 2 (see [24]). Let $\widetilde{\beta}_{i}=\left(\left[a_{i}, b_{i}, c_{i}, d_{i}\right] ;\left[\mu_{i}, \bar{\mu}_{i}\right],\left[\underline{v}_{i}, \bar{v}_{i}\right]\right)$ ( $i=1,2)$ be two IITFNs and $\lambda \geq 0$; then the following four equations hold:

(1) $\tilde{\beta}_{1}+\widetilde{\beta}_{2}=\left(\left[a_{1}+a_{2}, b_{1}+b_{2}, c_{1}+c_{2}, d_{1}+d_{2}\right] ;\left[\underline{\mu}_{1}+\underline{\mu}_{2}-\right.\right.$ $\left.\left.\underline{\mu}_{1} \underline{\mu}_{2}, \bar{\mu}_{1}+\bar{\mu}_{2}-\bar{\mu}_{1} \bar{\mu}_{2}\right],\left[\underline{v}_{1} \underline{v}_{2}, \bar{v}_{1} \bar{v}_{2}\right]\right)$

(2) $\tilde{\beta}_{1} \widetilde{\beta}_{2}=\left(\left[a_{1} a_{2}, b_{1} b_{2}, c_{1} c_{2}, d_{1} d_{2}\right] ;\left[\underline{\mu}_{1} \underline{\mu}_{2}, \bar{\mu}_{1} \bar{\mu}_{2}\right],\left[\underline{v}_{1}+\underline{v}_{2}-\right.\right.$ $\left.\left.\underline{v}_{1} \underline{v}_{2}, \bar{v}_{1}+\bar{v}_{2}-\bar{v}_{1} \bar{v}_{2}\right]\right)$

(3) $\lambda \widetilde{\beta}_{1}=\left(\left[\lambda a_{1}, \lambda b_{1}, \lambda c_{1}, \lambda d_{1}\right] ;\left[1-\left(1-\underline{\mu}_{1}\right)^{\lambda}, 1-\left(1-\bar{\mu}_{1}\right)^{\lambda}\right]\right.$, $\left.\left[\left(\underline{v}_{1}\right)^{\lambda},\left(\bar{v}_{1}\right)^{\lambda}\right]\right)$;

(4) $\widetilde{\beta}_{1}^{\lambda}=\left(\left[a_{1}^{\lambda}, b_{1}^{\lambda}, c_{1}^{\lambda}, d_{1}^{\lambda}\right] ;\left[\left(\underline{\mu}_{1}\right)^{\lambda},\left(\bar{\mu}_{1}\right)^{\lambda}\right]\left[1-\left(1-\underline{v}_{1}\right)^{\lambda}, 1-\right.\right.$ $\left.\left.\left(1-\bar{v}_{1}\right)^{\lambda}\right]\right)$.

Definition 3. Let $\widetilde{\beta}=([a, b, c, d] ;[\underline{\mu}, \bar{\mu}],[\underline{v}, \bar{v}])$ be an IITFN; then

$$
S(\widetilde{\beta})=\frac{a+2 b+2 c+d}{6} \cdot \frac{\underline{\mu}-\underline{v}+\bar{\mu}-\bar{v}}{2}
$$

is a score of $\widetilde{\beta}$, and $S$ is a score function, where $S(\widetilde{\beta}) \in$ $[-1,1]$. The larger the score $S(\widetilde{\beta})$ is, the greater the IITFN $\widetilde{\beta}$ is. Moreover,

$$
A(\tilde{\beta})=\frac{a+2 b+2 c+d}{6} \cdot \frac{\mu+\underline{v}+\bar{\mu}+\bar{v}}{2}
$$

is an accuracy degree of $\widetilde{\beta}$, and $A$ is an accuracy function, where $A(\widetilde{\beta}) \in[0,1]$. The larger the value of $A(\widetilde{\beta})$ is, the greater the accuracy degree of $\widetilde{\beta}$ is.

In the following, we introduce an order relation between any pair of IITFNs based on (3) and (4).

Definition 4. Let $\widetilde{\beta}_{i}=\left(\left[a_{i}, b_{i}, c_{i}, d_{i}\right] ;\left[\underline{\mu}_{i}, \bar{\mu}_{i}\right],\left[\underline{v}_{i}, \bar{v}_{i}\right]\right)(i=1,2)$ be two IITFNs; then

(i) if $S\left(\widetilde{\beta}_{1}\right)>S\left(\widetilde{\beta}_{2}\right)$, then $\widetilde{\beta}_{1}$ is larger than $\widetilde{\beta}_{2}$, denoted by $\widetilde{\beta}_{1}>\widetilde{\beta}_{2}$

(ii) if $S\left(\widetilde{\beta}_{1}\right)=S\left(\widetilde{\beta}_{2}\right)$, then

(1) if $A\left(\widetilde{\beta}_{1}\right)=A\left(\widetilde{\beta}_{2}\right)$, then $\widetilde{\beta}_{1}$ and $\widetilde{\beta}_{2}$ represent the same information, denoted by $\widetilde{\beta}_{1}=\widetilde{\beta}_{2}$; 
(2) if $A\left(\widetilde{\beta}_{1}\right)>A\left(\widetilde{\beta}_{2}\right)$, then $\widetilde{\beta}_{1}$ is larger than $\widetilde{\beta}_{2}$, denoted by $\widetilde{\beta}_{1}>\widetilde{\beta}_{2}$.

Definition 5 (see [24]). Let $\widetilde{\beta}_{j}=\left(\left[a_{j}, b_{j}, c_{j}, d_{j}\right] ;\left[\underline{\mu}_{j}, \bar{\mu}_{j}\right]\right.$, $\left.\left[\underline{v}_{j}, \bar{v}_{j}\right]\right)(j=1,2, \ldots, n)$ be a collection of IITFNs, and let IITFWG $\Psi^{n} \rightarrow \Psi$; if

$$
\begin{aligned}
& \operatorname{IITFWG}_{v}\left(\widetilde{\beta}_{1}, \widetilde{\beta}_{2}, \ldots, \widetilde{\beta}_{n}\right) \\
&=\prod_{j=1}^{n}\left(\widetilde{\beta}_{j}\right)^{v_{j}} \\
&=\left(\left[\prod_{j=1}^{n}\left(a_{j}\right)^{v_{j}}, \prod_{j=1}^{n}\left(b_{j}\right)^{v_{j}}, \prod_{j=1}^{n}\left(c_{j}\right)^{v_{j}}, \prod_{j=1}^{n}\left(d_{j}\right)^{v_{j}}\right] ;\right. \\
& {\left[\prod_{j=1}^{n}\left(\underline{\mu}_{j}\right)^{v_{j}} \prod_{j=1}^{n}\left(\bar{\mu}_{j}\right)^{v_{j}}\right], } \\
& {\left.\left[1-\prod_{j=1}^{n}\left(1-\underline{v}_{j}\right)^{v_{j}}, 1-\prod_{j=1}^{n}\left(1-\bar{v}_{j}\right)^{v_{j}}\right]\right), }
\end{aligned}
$$

then IITFWG is called the interval intuitionistic trapezoidal fuzzy weighted geometric (IITFWG) operator of dimension $n$, where $v=\left\{v_{1}, v_{2}, \ldots, v_{n}\right\}^{T}$ is the weight vector of $\widetilde{\beta}_{j}(j=$ $1,2, \ldots, n)$, with $v_{j}>0$ and $\sum_{j=1}^{n} v_{j}=1$.

Definition 6 (see [26]). Let $\widetilde{\beta}_{j}=\left(\left[a_{j}, b_{j}, c_{j}, d_{j}\right] ;\left[\underline{\mu}_{j}, \bar{\mu}_{j}\right]\right.$, $\left.\left[\underline{v}_{j}, \bar{v}_{j}\right]\right)(j=1,2, \ldots, n)$ be a collection of IITFNs, and let IITFOWG $Q^{n} \rightarrow Q$, if

$$
\begin{aligned}
& \text { IITFOWG }_{\varepsilon}\left(\widetilde{\beta}_{1}, \widetilde{\beta}_{2}, \ldots, \widetilde{\beta}_{n}\right) \\
&=\prod_{j=1}^{n}\left(\widetilde{\beta}_{\sigma(j)}\right)^{\varepsilon_{j}} \\
&=\left(\left[\prod_{j=1}^{n}\left(a_{\sigma(j)}\right)^{\varepsilon_{j}}, \prod_{j=1}^{n}\left(b_{\sigma(j)}\right)^{\varepsilon_{j}}, \prod_{j=1}^{n}\left(c_{\sigma(j)}\right)^{\varepsilon_{j}}, \prod_{j=1}^{n}\left(d_{\sigma(j)}\right)^{\varepsilon_{j}}\right] ;\right. \\
& {\left[\prod_{j=1}^{n}\left(\underline{\mu}_{\sigma(j)}\right)^{\varepsilon_{j}} \prod_{j=1}^{n}\left(\bar{\mu}_{\sigma(j)}\right)^{\varepsilon_{j}}\right], } \\
& {\left.\left[1-\prod_{j=1}^{n}\left(1-\underline{v}_{\sigma(j)}\right)^{\varepsilon_{j}}, 1-\prod_{j=1}^{n}\left(1-\bar{v}_{\sigma(j)}\right)^{\varepsilon_{j}}\right]\right) ; }
\end{aligned}
$$

then IITFOWG is called the interval intuitionistic trapezoidal fuzzy ordered weighted geometric (IITFOWG) operator, where $(\sigma(1), \sigma(2), \ldots, \sigma(n))$ is a permutation of $(1,2, \ldots, n)$, such that $\widetilde{\beta}_{\sigma(j-1)} \geq \widetilde{\beta}_{\sigma(j)}$ for all $j=1,2, \ldots, n$, with $\varepsilon_{j}>0$ and $\sum_{j=1}^{n} \varepsilon_{j}=1$.
Definition 7 (see [25]). Let $\widetilde{\beta}_{i}=\left(\left[a_{i}, b_{i}, c_{i}, d_{i}\right] ;\left[\underline{\mu}_{i}, \bar{\mu}_{i}\right],\left[\underline{v}_{i}, \bar{v}_{i}\right]\right)$ $(i=1,2)$ be two IITFNs; then the Hamming distance between $\widetilde{\beta}_{1}$ and $\widetilde{\beta}_{2}$ can be defined as

$$
\begin{aligned}
d\left(\widetilde{\beta}_{1}, \widetilde{\beta}_{2}\right)= & {\left[\left|\left(\underline{\mu}_{1}-\bar{v}_{1}\right) a_{1}-\left(\underline{\mu}_{2}-\bar{v}_{2}\right) a_{2}\right|\right.} \\
& +\left|\left(\bar{\mu}_{1}-\underline{v}_{1}\right) a_{1}-\left(\bar{\mu}_{2}-\underline{v}_{2}\right) a_{2}\right| \\
& +\left|\left(\underline{\mu}_{1}-\bar{v}_{1}\right) b_{1}-\left(\underline{\mu}_{2}-\bar{v}_{2}\right) b_{2}\right| \\
& +\left|\left(\bar{\mu}_{1}-\underline{v}_{1}\right) b_{1}-\left(\bar{\mu}_{2}-\underline{v}_{2}\right) b_{2}\right| \\
& +\left|\left(\underline{\mu}_{1}-\bar{v}_{1}\right) c_{1}-\left(\underline{\mu}_{2}-\bar{v}_{2}\right) c_{2}\right| \\
& +\left|\left(\bar{\mu}_{1}-\underline{v}_{1}\right) c_{1}-\left(\bar{\mu}_{2}-\underline{v}_{2}\right) c_{2}\right| \\
& +\left|\left(\underline{\mu}_{1}-\bar{v}_{1}\right) d_{1}-\left(\underline{\mu}_{2}-\bar{v}_{2}\right) d_{2}\right| \\
& \left.+\left|\left(\bar{\mu}_{1}-\underline{v}_{1}\right) d_{1}-\left(\bar{\mu}_{2}-\underline{v}_{2}\right) d_{2}\right|\right] / 8 .
\end{aligned}
$$

Definition 8. Let $Y=\left(\widetilde{y}_{i j}\right)_{m \times n}$ be a matrix. If all $\widetilde{y}_{i j}$ are IITFNs and $\tilde{y}_{i j}=\left(\left[a_{i j}, b_{i j}, c_{i j}, d_{i j}\right] ;\left[\underline{\mu}_{i j}, \bar{\mu}_{i j}\right],\left[\underline{v}_{i j}, \bar{v}_{i j}\right]\right), i=1,2, \ldots, m$, $j=1,2, \ldots, n$, then we call $Y$ an interval intuitionistic trapezoidal fuzzy matrix (IITFM). Similar to (7), the distance measure of two IITFMs $Y_{1}$ and $Y_{2}$ is defined as

$$
\begin{aligned}
d\left(Y_{1}, Y_{2}\right) & \\
=\frac{1}{8 m n} \sum_{i=1}^{m} \sum_{j=1}^{n} & \left(\left|\left(\underline{\mu}_{i j}^{(1)}-\bar{v}_{i j}^{(1)}\right) a_{i j}^{(1)}-\left(\underline{\mu}_{i j}^{(2)}-\bar{v}_{i j}^{(2)}\right) a_{i j}^{(2)}\right|\right. \\
& +\left|\left(\bar{\mu}_{i j}^{(1)}-\underline{v}_{i j}^{(1)}\right) a_{i j}^{(1)}-\left(\bar{\mu}_{i j}^{(2)}-\underline{v}_{i j}^{(2)}\right) a_{i j}^{(2)}\right| \\
& +\left|\left(\underline{\mu}_{i j}^{(1)}-\bar{v}_{i j}^{(1)}\right) b_{i j}^{(1)}-\left(\underline{\mu}_{i j}^{(2)}-\bar{v}_{i j}^{(2)}\right) b_{i j}^{(2)}\right| \\
& +\left|\left(\bar{\mu}_{i j}^{(1)}-\underline{v}_{i j}^{(1)}\right) b_{i j}^{(1)}-\left(\bar{\mu}_{i j}^{(2)}-\underline{v}_{i j}^{(2)}\right) b_{i j}^{(2)}\right| \\
& +\left|\left(\underline{\mu}_{i j}^{(1)}-\bar{v}_{i j}^{(1)}\right) c_{i j}^{(1)}-\left(\underline{\mu}_{i j}^{(2)}-\bar{v}_{i j}^{(2)}\right) c_{i j}^{(2)}\right| \\
& +\left|\left(\bar{\mu}_{i j}^{(1)}-\underline{v}_{i j}^{(1)}\right) c_{i j}^{(1)}-\left(\bar{\mu}_{i j}^{(2)}-\underline{v}_{i j}^{(2)}\right) c_{i j}^{(2)}\right| \\
& +\left|\left(\underline{\mu}_{i j}^{(1)}-\bar{v}_{i j}^{(1)}\right) d_{i j}^{(1)}-\left(\underline{\mu}_{i j}^{(2)}-\bar{v}_{i j}^{(2)}\right) d_{i j}^{(2)}\right| \\
& \left.+\left|\left(\bar{\mu}_{i j}^{(1)}-\underline{v}_{i j}^{(1)}\right) d_{i j}^{(1)}-\left(\bar{\mu}_{i j}^{(2)}-\underline{v}_{i j}^{(2)}\right) d_{i j}^{(2)}\right|\right),
\end{aligned}
$$

where $Y_{1}=\left(\widetilde{y}_{i j}^{(1)}\right)_{m \times n}=\left(\left(\left[a_{i j}^{(1)}, b_{i j}^{(1)}, c_{i j}^{(1)}, d_{i j}^{(1)}\right] ;\left[\underline{\mu}_{i j}^{(1)}, \bar{\mu}_{i j}^{(1)}\right]\right.\right.$, $\left.\left.\left[\underline{v}_{i j}^{(1)}, \bar{v}_{i j}^{(1)}\right]\right)\right)_{m \times n}$ and $Y_{2}=\left(\tilde{y}_{i j}^{(2)}\right)_{m \times n}=\left(\left(\left[a_{i j}^{(2)}, b_{i j}^{(2)}, c_{i j}^{(2)}, d_{i j}^{(2)}\right]\right.\right.$; $\left.\left.\left[\underline{\mu}_{i j}^{(2)}, \bar{\mu}_{i j}^{(2)}\right],\left[\underline{v}_{i j}^{(2)}, \bar{v}_{i j}^{(2)}\right]\right)\right)_{m \times n}$. 


\section{Multiattribute Group Decision Making Method Based on Interval Intuitionistic Trapezoidal Fuzzy Numbers}

In this section, we propose a new multiattribute interval intuitionistic trapezoidal fuzzy group decision making methodology based on distance measure, where the decision makers' weights are unknown.

Let $D=\left\{D_{1}, D_{2}, \ldots, D_{t}\right\}$ be a discrete set of decision makers in the group decision making, and let $\varepsilon=$ $\left(\varepsilon_{1}, \varepsilon_{2}, \ldots, \varepsilon_{t}\right)^{T}$ be the weight vector of decision makers, with $\varepsilon_{k} \in[0,1]$ and $\sum_{k=1}^{t} \varepsilon_{k}=1$. Let $A=\left\{A_{1}, A_{2}, \ldots, A_{m}\right\}$ be a finite set of alternatives, let $c=\left\{c_{1}, c_{2}, \ldots, c_{n}\right\}$ be a set of attributes, and let $v=\left(v_{1}, v_{2}, \ldots, v_{n}\right)^{T}$ be the weight vector of attributes, with $v_{j} \in[0,1]$ and $\sum_{j=1}^{n} v_{j}=$ 1. Suppose that $\widetilde{G}_{k}=\left(\widetilde{g}_{i j}^{(k)}\right)_{m \times n}$ is the interval intuitionistic trapezoidal fuzzy decision matrix, where $\widetilde{g}_{i j}^{(k)}=$ $\left(\left[x_{1 i}^{(k)}\left(c_{j}\right), x_{2 i}^{(k)}\left(c_{j}\right), x_{3 i}^{(k)}\left(c_{j}\right), x_{4 i}^{(k)}\left(c_{j}\right)\right] ;\left[\tilde{\mu}_{i j}^{(k)}, \widetilde{v}_{i j}^{(k)}\right]\right)$ is an IITFN, provided by the decision maker $D_{k} \in D$ for the alternatives $A_{i} \in A$ with respect to attribute $c_{j} \in c . \tilde{\mu}_{i j}^{k}$ indicates the degree to which alternative $A_{i}$ should satisfy the attribute $c_{j}$ expressed by expert $D_{k}$, while $\widetilde{v}_{i j}^{k}$ indicates the degree to which alternative $A_{i}$ should not satisfy the attribute $c_{j}$ given by expert $D_{k} ; \tilde{\mu}_{i j}^{(k)}=\left[\underline{\mu}_{i j}^{(k)}, \bar{\mu}_{i j}^{(k)}\right] \subseteq[0,1], \widetilde{v}_{i j}^{(k)}=\left[\underline{v}_{i j}^{(k)}, \bar{v}_{i j}^{(k)}\right] \subseteq$ $[0,1]$, and $0 \leq \sup \widetilde{\mu}_{i j}^{(k)}+\sup \widetilde{v}_{i j}^{(k)} \leq 1, i=1,2, \ldots, m$, $j=1,2, \ldots, n$, and $k=1,2, \ldots, t$.
In the following, we apply distance measure, IITFWG operator, and IITFOWG operator to multiattribute group decision making based on interval intuitionistic trapezoidal fuzzy information. The proposed method involves the following steps.

Step 1. Determine the weights of the decision makers.

First, normalize the fuzzy decision matrix.

It converts the fuzzy decision matrix $\widetilde{G}_{k}=\left(\widetilde{g}_{i j}^{(k)}\right)_{m \times n}$ into normalized decision matrix $\widetilde{E}_{k}=\left(\widetilde{e}_{i j}^{(k)}\right)_{m \times n}$, where $\widetilde{e}_{i j}^{(k)}=$ $\left(\left[e_{1 i}^{(k)}\left(c_{j}\right), e_{2 i}^{(k)}\left(c_{j}\right), e_{3 i}^{(k)}\left(c_{j}\right), e_{4 i}^{(k)}\left(c_{j}\right)\right] ;\left[\widetilde{\mu}_{i j}^{(k)}, \widetilde{v}_{i j}^{(k)}\right]\right)$.

And the normalized values for benefit-related criteria $(B)$ and cost-related criteria $(C)$ are calculated as follows:

$$
\begin{array}{r}
e_{l i}^{(k)}\left(c_{j}\right)=\frac{x_{l i}^{(k)}\left(c_{j}\right)-\min _{i}\left(x_{1 i}^{(k)}\left(c_{j}\right)\right)}{\max _{i}\left(x_{4 i}^{(k)}\left(c_{j}\right)\right)-\min _{i}\left(x_{1 i}^{(k)}\left(c_{j}\right)\right)}, \\
l=1,2,3,4, \quad j \in B, \\
e_{l i}^{(k)}\left(c_{j}\right)=\frac{\max _{i}\left(x_{4 i}^{(k)}\left(c_{j}\right)\right)-x_{5-l, i}^{(k)}\left(c_{j}\right)}{\max _{i}\left(x_{4 i}^{(k)}\left(c_{j}\right)\right)-\min _{i}\left(x_{1 i}^{(k)}\left(c_{j}\right)\right)}, \\
l=1,2,3,4, \quad j \in C .
\end{array}
$$

Second, determine the extreme matrixes $\widetilde{E}^{+}=\left(\widetilde{e}_{i j}^{+}\right)_{m \times n}$, $\widetilde{E}^{-}=\left(\widetilde{e_{i j}}\right)_{m \times n}$ and the average matrix $\widetilde{E}^{*}=\left(\widetilde{e}_{i j}^{*}\right)_{m \times n}$, shown as follows:

$$
\begin{gathered}
\tilde{e}_{i j}^{+}=\left(\left[\max _{1 \leq k \leq t} e_{1 i}^{(k)}\left(c_{j}\right), \max _{\substack{1 \leq k \leq t \\
1 \leq i \leq m}} e_{2 i}^{(k)}\left(c_{j}\right), \max _{\substack{1 \leq k \leq t \\
1 \leq i \leq m}} e_{3 i}^{(k)}\left(c_{j}\right), \max _{\substack{1 \leq k \leq t \\
1 \leq i \leq m}} e_{4 i}^{(k)}\left(c_{j}\right)\right] ;[1,1],[0,0]\right), \\
\widetilde{e}_{i j}^{-}=\left(\left[\min _{1 \leq k \leq t} e_{1 i}^{(k)}\left(c_{j}\right), \min _{\substack{1 \leq k \leq t \\
1 \leq i \leq m}} e_{2 i}^{(k)}\left(c_{j}\right), \min _{\substack{1 \leq k \leq t \\
1 \leq i \leq m}} e_{3 i}^{(k)}\left(c_{j}\right), \min _{\substack{1 \leq k \leq t \\
1 \leq i \leq m}} e_{4 i}^{(k)}\left(c_{j}\right)\right] ;[0,0],[1,1]\right), \\
\widetilde{e}_{i j}^{*}=\frac{1}{t}\left(e_{i j}^{(1)}+e_{i j}^{(2)}+\cdots+e_{i j}^{(t)}\right),
\end{gathered}
$$

where $i=1,2, \ldots, m, j=1,2, \ldots, n$, and $t$ is the total number of decision makers.

Third, calculate the distance measure of individual matrix $\widetilde{E}_{k}$ to extreme matrixes and average matrix, respectively, shown as follows:

$$
\begin{array}{ll}
S_{k}^{+}=S\left(\widetilde{E}_{k}, \widetilde{E}^{+}\right)=\sum_{i=1}^{m} \sum_{j=1}^{n} d\left(\widetilde{e}_{i j}^{(k)}, \widetilde{e}_{i j}^{+}\right), & k=1,2, \ldots, t, \\
S_{k}^{-}=S\left(\widetilde{E}_{k}, \widetilde{E}^{-}\right)=\sum_{i=1}^{m} \sum_{j=1}^{n} d\left(\widetilde{e}_{i j}^{(k)}, \widetilde{e}_{i j}\right), & k=1,2, \ldots, t, \\
S_{k}^{*}=S\left(\widetilde{E}_{k}, \widetilde{E}^{*}\right)=\sum_{i=1}^{m} \sum_{j=1}^{n} d\left(\widetilde{e}_{i j}^{(k)}, \widetilde{e}_{i j}^{*}\right), & k=1,2, \ldots, t .
\end{array}
$$

The average matrix reflects the group opinion, and the extreme matrixes reflect the extreme views of the experts. It is clear that the closer a decision matrix $\widetilde{E}_{k}$ is to the average matrix $\widetilde{E}^{*}$, the better the decision $\widetilde{E}_{k}$ of $k$ th $(k=1,2, \ldots, t)$ decision maker is, so the heavier weight should be given. At the same time, the closer a decision matrix $\widetilde{E}_{k}$ is to the extreme matrixes, the worse the decision of $k$ th expert is, so the smaller weight should be given. Based on the above analysis, the weight of the $k$ th decision maker is defined as follows:

$$
\varepsilon_{k}=\frac{\varepsilon_{k}^{*}}{\sum_{k=1}^{t} \varepsilon_{k}^{*}}, \quad \varepsilon_{k}^{*}=\frac{S_{k}^{+}+S_{k}^{-}}{S_{k}^{*}} .
$$

Step 2. Calculate the weights of attributes. 
Assume that the weight vectors with regard to different group members are described by $v^{k}=\left\{v_{1}^{k}, v_{2}^{k}, \ldots, v_{n}^{k}\right\}^{T}$, $k=1,2, \ldots, t$. Then the weight vector of attributes $v=$ $\left\{v_{1}, v_{2}, \ldots, v_{n}\right\}^{T}$ is calculated by aggregating decision makers' weights, shown as follows:

$$
v_{j}=\sum_{k=1}^{t} \varepsilon_{k} v_{j}^{k}, \quad j=1,2, \ldots, n .
$$

Step 3. Utilize the IITFWG operator to aggregate the $i$ th line element of the interval intuitionistic trapezoidal fuzzy decision matrix $\widetilde{E}_{k}=\left(\widetilde{e}_{i j}^{(k)}\right)_{m \times n}$ to obtain the individual overall interval intuitionistic trapezoidal fuzzy values $\hat{e}_{i}^{(k)}$ of the alternative $A_{i}$, where $\widetilde{e}_{i}^{(k)}=\operatorname{IITFWG}_{v}\left(\widetilde{e}_{i 1}^{(k)}, \widetilde{e}_{i 2}^{(k)}, \ldots, \widetilde{e}_{i n}^{(k)}\right)=$ $\sum_{j=1}^{n} v_{j} \widetilde{e}_{i j}^{(k)}, i=1,2, \ldots, m, k=1,2, \ldots, t$, and $v=$ $\left(v_{1}, v_{2}, \ldots, v_{n}\right)^{T}$ is the weight vector of attributes.

Step 4. Utilize the IITFWOG operator to aggregate $\widetilde{e}_{i}^{(k)}$ and obtain the collective overall IITFNS $\widetilde{e}_{i}$, where $\widetilde{e}_{i}=$ $\operatorname{IITFWOG}_{\varepsilon, v}\left(\widetilde{e}_{i}^{(1)}, \widetilde{e}_{i}^{(2)}, \ldots, \widetilde{e}_{i}^{(t)}\right)$ and $\varepsilon=\left(\varepsilon_{1}, \varepsilon_{2}, \ldots, \varepsilon_{t}\right)^{T}$ is the weight vector of decision makers.

Step 5. Rank the preference order of all alternatives according to the score function and accuracy function.

\section{Illustrative Example}

In this section, we present an illustrative example of emergency alternatives selection to demonstrate the potential application of the proposed method.

To implement the rescue action of a certain urban fire happened in a Cotton Mill, an emergency department is desired to select the most suitable emergency alternatives from three preevaluation emergency alternatives: $\left\{A_{1}, A_{2}, A_{3}\right\}$. Three criteria are considered as follows: cost of consumption $\left(c_{1}\right)$, negative impact of the rescue $\left(c_{2}\right)$, and disposal time $\left(c_{3}\right)$. In order to select the best alternative, a committee composed of three experts $D_{k}(k=1,2,3)$ has been found. The three alternatives are to be evaluated using the IITFNs by the three experts under the above three criteria, as listed in Table 1.

In the following, we utilize the proposed method to select the most desirable emergency alternatives.

Step 1. Calculate the decision expert weights vector.

First, by applying (9), the fuzzy decision matrix is normalized, which is shown in Table 2.

Furthermore, based on (10)-(11), we can get the extreme matrixes $\left(\widetilde{E}^{+}, \widetilde{E}^{-}\right)$, average matrix $\left(\widetilde{E}^{*}\right)$, and the distance measure values, which are shown as follows:

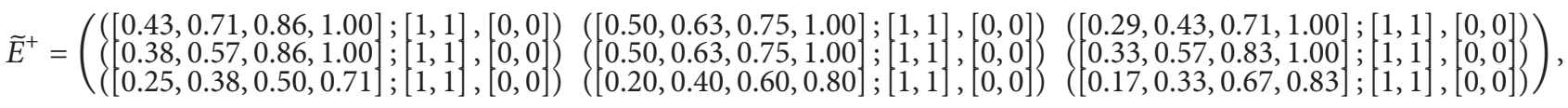

$$
\begin{aligned}
& \widetilde{E}^{-}=\left(\begin{array}{cccc}
([0.00,0.38,0.63,0.88] ;[0,0],[1,1]) & ([0.13,0.38,0.50,0.75] ;[0,0],[1,1]) & ([0.00,0.17,0.33,0.67] ;[0,0],[1,1]) \\
([0.13,0.50,0.63,1.00] ;[0,0],[1,1]) & ([0.00,0.20,0.40,0.60] ;[0,0],[1,1]) & ([0.14,0.50,0.71,1.00] ;[0,0],[1,1]) \\
([0.00,0.29,0.38,0.50] ;[0,0],[1,1]) & ([0.00,0.13,0.25,0.38] ;[0,0],[1,1]) & ([0.00,0.17,0.17,0.17] ;[0,0],[1,1])
\end{array}\right. \\
& \widetilde{E}^{*}=\left(\begin{array}{lll}
([0.23,0.49,0.71,0.92] ; & ([0.28,0.47,0.68,0.88] ; & ([0.10,0.26,0.51,0.78] ; \\
[0.38,0.40],[0.14,0.33]) & [0.40,0.60],[0.23,0.37]) & [0.40,0.55],[0.16,0.33]) \\
([0.27,0.52,0.75,1.00] ; & ([0.25,0.40,0.63,0.87] ; & ([0.27,0.52,0.79,1.00] ; \\
[0.10,0.34],[0.33,0.56]) & [0.27,0.50],[0.40,0.50]) & [0.30,0.50],[0.30,0.48]) \\
([0.08,0.27,0.44,0.61] ; & ([0.07,0.26,0.45,0.60] ; & ([0.06,0.26,0.42,0.57] ; \\
[0.35,0.52],[0.20,0.36]) & [0.40,0.60],[0.27,0.40]) & [0.63,0.70],[0.16,0.23])
\end{array}\right)
\end{aligned}
$$

$S_{1}^{+}=0.57, S_{1}^{-}=0.42, S_{1}^{*}=0.268 ; S_{2}^{+}=0.616, S_{2}^{-}=0.362$, $S_{2}^{*}=0.08 ; S_{3}^{+}=0.59, S_{3}^{-}=0.396$, and $S_{3}^{*}=0.089$.

Finally, using the distance measure equation (12), we can determine the weight vector of decision makers, which is $\varepsilon=$ $(0.14,0.45,0.41)^{T}$.

Step 2. Calculate the weights vector of the attributes.

The weight vector of the three criteria from each decision maker is as follows: $v^{1}=(0.2,0.3,0.5)^{T}, v^{2}=(0.1,0.5,0.4)^{T}$, and $v^{3}=(0.2,0.4,0.4)^{T}$. Then, from (13), we can acquire the weight vector of attributes, which is $v=(0.16,0.43,0.41)^{T}$.

Step 3. Determine the individual overall IITFNs.
By using the IITFWG operator and the normalized fuzzy decision matrix $\widetilde{E}_{k}$, we can calculate the individual overall IITFNs $\widetilde{e}_{i}^{(k)}(i=1,2,3, k=1,2,3)$, shown as follows:

$$
\begin{aligned}
& \widetilde{e}_{1}^{(1)}=([0.26,0.45,0.77,1.00] ;[0.27,0.48],[0.24,0.39]), \\
& \widetilde{e}_{2}^{(1)}=([0.00,0.36,0.57,0.80] ;[0.29,0.60],[0.26,0.40]), \\
& \widetilde{e}_{3}^{(1)}=([0.00,0.33,0.50,0.75] ;[0.36,0.46],[0.24,0.42]), \\
& \widetilde{e}_{1}^{(2)}=([0.00,0.34,0.52,0.79] ;[0.32,0.44],[0.28,0.46]), \\
& \widetilde{e}_{2}^{(2)}=([0.30,0.44,0.76,1.00] ;[0.24,0.34],[0.43,0.54]), \\
& \widetilde{e}_{3}^{(2)}=([0.00,0.25,0.54,0.68] ;[0.49,0.68],[0.18,0.28]),
\end{aligned}
$$


TABLE 1: The decision matrix with IITFNs for each decision maker.

\begin{tabular}{clccc}
\hline$D_{k}$ & $A_{i}$ & Cost $c_{1}$ & Impact $c_{2}$ & Time $c_{3}$ \\
\hline \multirow{2}{*}{$D_{1}$} & $A_{1}$ & $([1,2,3,4] ;[0.4,0.7],[0.1,0.3])$ & $([1,2,4,5] ;[0.3,0.5],[0.4,0.5])$ & $([2,4,6,7] ;[0.2,0.4],[0.1,0.3])$ \\
& $A_{2}$ & $([1,2,4,6] ;[0.2,0.4],[0.3,0.6])$ & $([3,4,5,6] ;[0.2,0.6],[0.3,0.4])$ & $([2,4,5,8] ;[0.5,0.7],[0.2,0.3])$ \\
& $A_{3}$ & $([3,5,6,8] ;[0.2,0.3],[0.4,0.6])$ & $([2,3,4,5] ;[0.4,0.5],[0.3,0.5])$ & $([4,6,7,9] ;[0.4,0.5],[0.1,0.2])$ \\
\hline \multirow{3}{*}{$D_{2}$} & $A_{1}$ & $([1,3,6,7] ;[0.2,0.4],[0.4,0.5])$ & $([2,3,4,5] ;[0.4,0.5],[0.3,0.5])$ & $([4,6,7,8] ;[0.3,0.5],[0.2,0.4])$ \\
& $A_{2}$ & $([1,3,5,6] ;[0.2,0.3],[0.3,0.5])$ & $([1,3,6,7] ;[0.3,0.4],[0.4,0.5])$ & $([2,3,5,6] ;[0.2,0.3],[0.5,0.6])$ \\
& $A_{3}$ & $([5,6,8,9] ;[0.5,0.6],[0.1,0.2])$ & $([4,5,7,9] ;[0.4,0.7],[0.2,0.3])$ & $([3,4,6,8] ;[0.6,0.7],[0.2,0.3])$ \\
\hline & $A_{1}$ & $([2,4,6,9] ;[0.5,0.6],[0.1,0.2])$ & $([3,4,5,8] ;[0.5,0.7],[0.1,0.2])$ & $([4,5,7,8] ;[0.6,0.7],[0.2,0.3])$ \\
$D_{3}$ & $A_{2}$ & $([1,3,5,8] ;[0.0,0.3],[0.4,0.6])$ & $([1,3,4,5] ;[0.3,0.4],[0.5,0.6])$ & $([2,3,5,6] ;[0.1,0.4],[0.3,0.6])$ \\
& $A_{3}$ & $([4,5,6,7] ;[0.3,0.6],[0.2,0.4])$ & $([6,7,8,9] ;[0.4,0.6],[0.3,0.4])$ & $([7,7,7,7] ;[0.8,0.8],[0.2,0.2])$ \\
\hline
\end{tabular}

TABLE 2: Normalized fuzzy decision matrix $\widetilde{E}_{k}$.

\begin{tabular}{cccccc}
\hline$D_{k}$ & $A_{i}$ & Cost $c_{1}$ & Impact $c_{2}$ & Time $c_{3}$ \\
\hline & $A_{1}$ & $([0.43,0.71,0.86,1.00] ;[0.4,0.7],[0.1,0.3])$ & $([0.20,0.40,0.80,1.00] ;[0.3,0.5],[0.4,0.5])$ & $([0.29,0.43,0.71,1.00] ;[0.2,0.4],[0.1,0.3])$ \\
$D_{1}$ & $A_{2}$ & $([0.29,0.57,0.86,1.00] ;[0.2,0.4],[0.3,0.6])$ & $([0.00,0.20,0.40,0.60] ;[0.2,0.6],[0.3,0.4])$ & $([0.14,0.57,0.71,1.00] ;[0.5,0.7],[0.2,0.3])$ \\
& $A_{3}$ & $([0.00,0.29,0.43,0.71] ;[0.2,0.3],[0.4,0.6])$ & $([0.20,0.40,0.60,0.80] ;[0.4,0.5],[0.3,0.5])$ & $([0.00,0.29,0.43,0.71] ;[0.4,0.5],[0.1,0.2])$ \\
\hline & $A_{1}$ & $([0.25,0.38,0.63,0.88] ;[0.2,0.4],[0.4,0.5])$ & $([0.50,0.63,0.75,0.88] ;[0.4,0.5],[0.3,0.5])$ & $([0.00,0.17,0.33,0.67] ;[0.3,0.5],[0.2,0.4])$ \\
$D_{2}$ & $A_{2}$ & $([0.38,0.50,0.63,1.00] ;[0.2,0.3],[0.3,0.5])$ & $([0.25,0.38,0.75,1.00] ;[0.3,0.4],[0.4,0.5])$ & $([0.33,0.50,0.83,1.00] ;[0.2,0.3],[0.5,0.6])$ \\
& $A_{3}$ & $([0.00,0.13,0.38,0.50] ;[0.5,0.6],[0.1,0.2])$ & $([0.00,0.25,0.50,0.63] ;[0.4,0.7],[0.2,0.3])$ & $([0.00,0.33,0.67,0.83] ;[0.6,0.7],[0.2,0.3])$ \\
\hline & $A_{1}$ & $([0.00,0.38,0.63,0.88] ;[0.5,0.6],[0.1,0.2])$ & $([0.13,0.38,0.50,0.75] ;[0.5,0.7],[0.1,0.2])$ & $([0.00,0.17,0.50,0.67] ;[0.6,0.7],[0.2,0.3])$ \\
$D_{3}$ & $A_{2}$ & $([0.13,0.50,0.75,1.00] ;[0.0,0.3],[0.4,0.6])$ & $([0.50,0.63,0.75,1.00] ;[0.30 .4],[0.5,0.6])$ & $([0.33,0.50,0.83,1.00] ;[0.1,0.4],[0.3,0.6])$ \\
& $A_{3}$ & $([0.25,0.38,0.50,0.63] ;[0.3,0.6],[0.2,0.4])$ & $([0.00,0.13,0.25,0.38] ;[0.4,0.6],[0.3,0.4])$ & $([0.17,0.17,0.17,0.17] ;[0.8,0.8],[0.2,0.2])$ \\
\hline
\end{tabular}

TABLE 3: Overall IITFNs, score degrees, and ranking of alternatives.

\begin{tabular}{lcc}
\hline Alternatives & Overall IITFNs & Score degrees \\
\hline$A_{1}$ & $([0.00,0.32,0.55,0.79] ;[0.32,0.49],[0.21,0.34])$ & 0.055 \\
$A_{2}$ & $([0.00,0.42,0.68,0.91] ;[0.00,0.39],[0.36,0.50])$ & -0.1218 \\
$A_{3}$ & $([0.00,0.22,0.37,0.49] ;[0.45,0.58],[0.22,0.32])$ & 0.068 \\
\hline
\end{tabular}

$$
\begin{aligned}
& \widetilde{e}_{1}^{(3)}=([0.00,0.27,0.52,0.73] ;[0.54,0.68],[0.14,0.24]), \\
& \widetilde{e}_{2}^{(3)}=([0.34,0.55,0.78,1.00] ;[0.00,0.38],[0.41,0.60]) \\
& \widetilde{e}_{3}^{(3)}=([0.00,0.17,0.24,0.30] ;[0.51,0.68],[0.24,0.32])
\end{aligned}
$$

Step 4. Determine the collective overall IITFNs and rank the alternatives.

By using the IITFWOG operator, we can obtain the collective overall IITFNS $\widetilde{e}_{i}(i=1,2,3)$, and based on (3) and (4), we can calculate the score degree of the overall IITFNs, which is shown in Table 3.

The final group ranking of the three alternatives can thus be obtained as $A_{3}>A_{1}>A_{2}$, and the most desirable alternative is $A_{3}$.

\section{Concluding Remarks}

In this paper, concerning the multiattribute group decision making problems in which the preference values are presented in the form of interval intuitionistic trapezoidal fuzzy numbers and the decision expert weights are unknown, a new analyzing method of decision making is proposed. By employing distance measure between individual matrix and extreme matrix, as well as the average matrix, the decision makers' weights have been measured. The proposed method can better reflect and describe the essential characteristic of the objective world and provide a more accurate, effective, and systematic decision support tool. In addition, an illustrative example of emergency alternative selection problem is provided to illustrate the practicality and effectiveness of the proposed methodology. Further research is to carry out sensitivity analyses and may continue extending the developed method to other domains.

\section{Conflict of Interests}

The authors declare that there is no conflict of interests regarding the publication of this paper.

\section{Acknowledgments}

The research was supported by the Decision-Making Bidding Project of Henan Provincial Government (no. 2014427); the Foundation of Social Sciences \& Humanities of Education, Department of Henan Province (no. 2014-qn-103); the Henan Association of Social Science (no. SKL-2014-260); and the 
National Youth Natural Science Foundation of China (no. 11301141).

\section{References}

[1] K. T. Atanassov, "Intuitionistic fuzzy sets," Fuzzy Sets and Systems, vol. 20, no. 1, pp. 87-96, 1986.

[2] K. Atanassov and G. Gargov, "Interval valued intuitionistic fuzzy sets," Fuzzy Sets and Systems, vol. 31, no. 3, pp. 343-349, 1989.

[3] L. A. Zadeh, "Fuzzy sets," Information and Control, vol. 8, pp. 338-353, 1965.

[4] S. Chen, L. Lee, H. Liu, and S. Yang, "Multiattribute decision making based on interval-valued intuitionistic fuzzy values," Expert Systems with Applications, vol. 39, no. 12, pp. 10343-10351, 2012.

[5] G. Beliakov, H. Bustince, S. James et al., "Aggregation for Atanassov's intuitionistic and interval valued fuzzy sets: the median operator," IEEE Transactions on Fuzzy Systems, vol. 20, pp. 487-498, 2012.

[6] Z. Xu, "Intuitionistic fuzzy multiattribute decision making: an interactive method," IEEE Transactions on Fuzzy Systems, vol. 20, no. 3, pp. 514-525, 2012.

[7] Z. C. Jiang and L. Y. Yu, "A multiple attribute intervalvalued intuitionistic fuzzy group decision making method for emergency alternative selection," Journal of Computational Information Systems, vol. 9, no. 12, pp. 4911-4919, 2013.

[8] L.-H. Chen, C.-C. Hung, and C.-C. Tu, "Considering the decision maker's attitudinal character to solve multi-criteria decision-making problems in an intuitionistic fuzzy environment," Knowledge-Based Systems, vol. 36, pp. 129-138, 2012.

[9] D. Yu, Y. Wu, and T. Lu, "Interval-valued intuitionistic fuzzy prioritized operators and their application in group decision making," Knowledge-Based Systems, vol. 30, pp. 57-66, 2012.

[10] F. Meng, C. Tan, and Q. Zhang, "The induced generalized interval-valued intuitionistic fuzzy hybrid Shapley averaging operator and its application in decision making," KnowledgeBased Systems, vol. 42, pp. 9-19, 2013.

[11] J. Mao, D. Yao, and C. Wang, "A novel cross-entropy and entropy measures of IFSs and their applications," KnowledgeBased Systems, vol. 48, pp. 37-45, 2013.

[12] M. Shu, C. Cheng, and J. Chang, "Using intuitionistic fuzzy sets for fault-tree analysis on printed circuit board assembly," Microelectronics Reliability, vol. 46, no. 12, pp. 2139-2148, 2006.

[13] D. F. Li, "A ratio ranking method of triangular intuitionistic fuzzy numbers and its application to MADM problems," Computers \& Mathematics with Applications, vol. 60, no. 6, pp. 15571570, 2010.

[14] Y. Chen and B. Li, "Dynamic multi-attribute decision making model based on triangular intuitionistic fuzzy numbers," Scientia Iranica B, vol. 18, no. 2, pp. 268-274, 2011.

[15] J. Q. Wang, R. Nie, H. Zhang, and X. Chen, "Intuitionistic fuzzy multi-criteria decision-making method based on evidential reasoning," Applied Soft Computing Journal, vol. 13, no. 4, pp. 1823-1831, 2013.

[16] H. M. Nehi and H. R. Maleki, "Intuitionistic fuzzy numbers and it's applications in fuzzy optimization problem," in Proceedings of the 9th WSEAS International Conference on Systems, Athens, Greece, 2005.

[17] J. Q. Wang and Z. Zhong, "Aggregation operators on intuitionistic trapezoidal fuzzy number and its application to multi-criteria decision making problems," Journal of Systems Engineering and Electronics, vol. 20, no. 2, pp. 321-326, 2009.

[18] S. P. Wan, "Survey on intuitionistic fuzzy multi-attribute decision making approach," Control and Decision, vol. 25, no. 11, pp. 1601-1606, 2010.

[19] S. P. Wan and J. Y. Dong, "Method of the intuitionistic trapezoidal fuzzy number for multi-attribute group decision," Control and Decision, vol. 25, no. 5, pp. 773-776, 2010.

[20] J. Ye, "Expected value method for intuitionistic trapezoidal fuzzy multicriteria decision-making problems," Expert Systems with Applications, vol. 38, no. 9, pp. 11730-11734, 2011.

[21] J. Ye, "Multicriteria group decision-making method using the distances-based similarity measures between intuitionistic trapezoidal fuzzy numbers," International Journal of General Systems, vol. 41, no. 7, pp. 729-739, 2012.

[22] S. P. Wan, X. Zhang, and D. Li, "Possibility mean-variance based method for intuitionistic trapezoidal fuzzy group decision making," Journal of System Engineering, vol. 27, pp. 782-787, 2012.

[23] X. Zhang, F. Jin, and P. Liu, "A grey relational projection method for multi-attribute decision making based on intuitionistic trapezoidal fuzzy number," Applied Mathematical Modelling, vol. 37, no. 5, pp. 3467-3477, 2013.

[24] S. P. Wan, "Multi-attribute decision making method based on interval-valued intuitionistic trapezoidal fuzzy numbers," Control and Decision, vol. 26, no. 6, pp. 857-860, 2011.

[25] S. P. Wan, "Multi-attribute decision making method based on interval-valued trapezoidal intuitionistic fuzzy numbers," Control and Decision, vol. 27, no. 3, pp. 455-458, 2012.

[26] G. Wei, X. Zhao, and H. Wang, "An approach to multiple attribute group decision making with interval intuitionistic trapezoidal fuzzy information," Technological and Economic Development of Economy, vol. 18, no. 2, pp. 317-330, 2012.

[27] J. Q. Wang, "An overview of fuzzy multi-criteria decisionmaking approach," Control and Decision, vol. 23, no. 6, pp. 601606,2008 . 


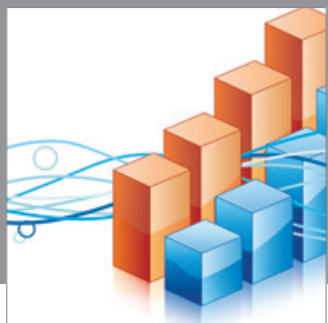

Advances in

Operations Research

mansans

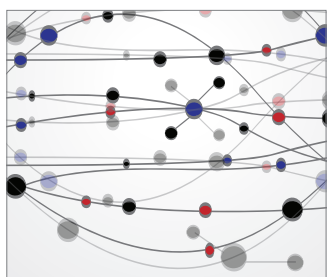

The Scientific World Journal
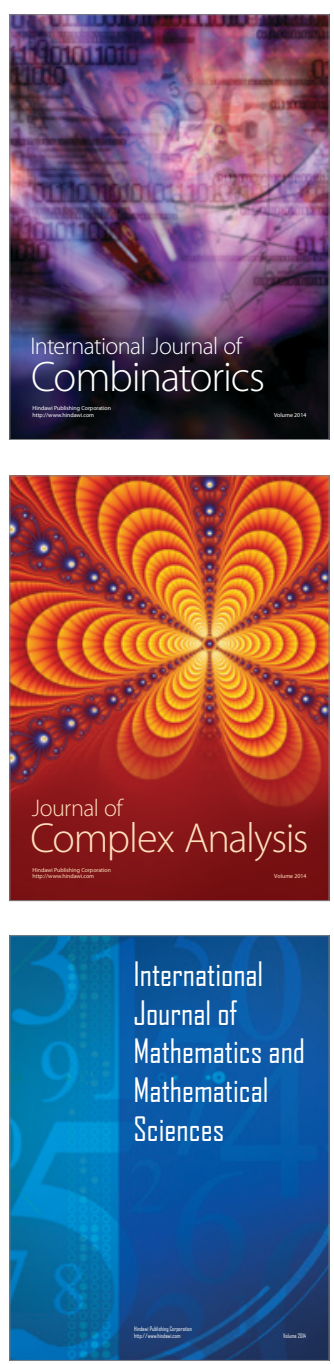
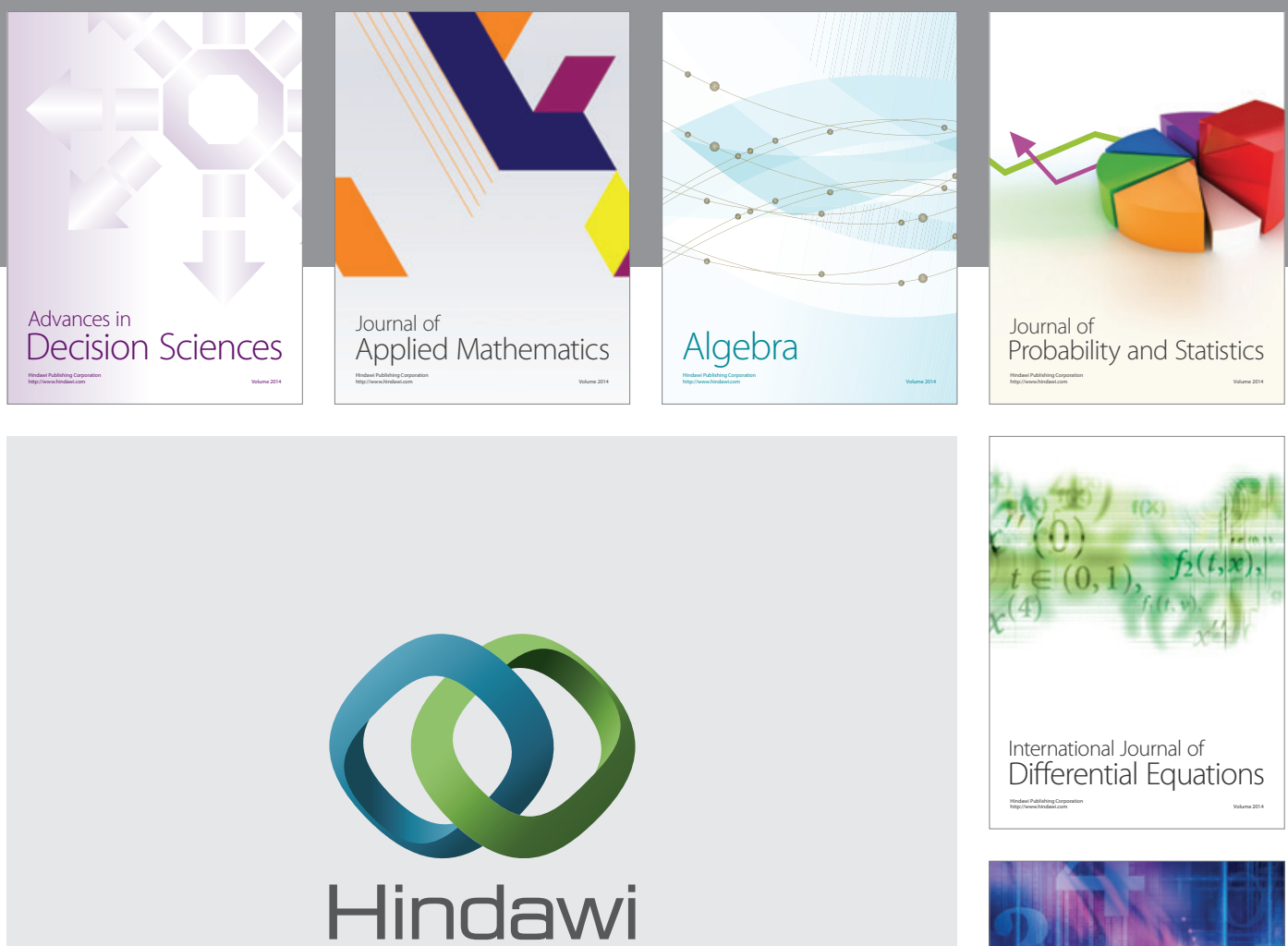

Submit your manuscripts at http://www.hindawi.com
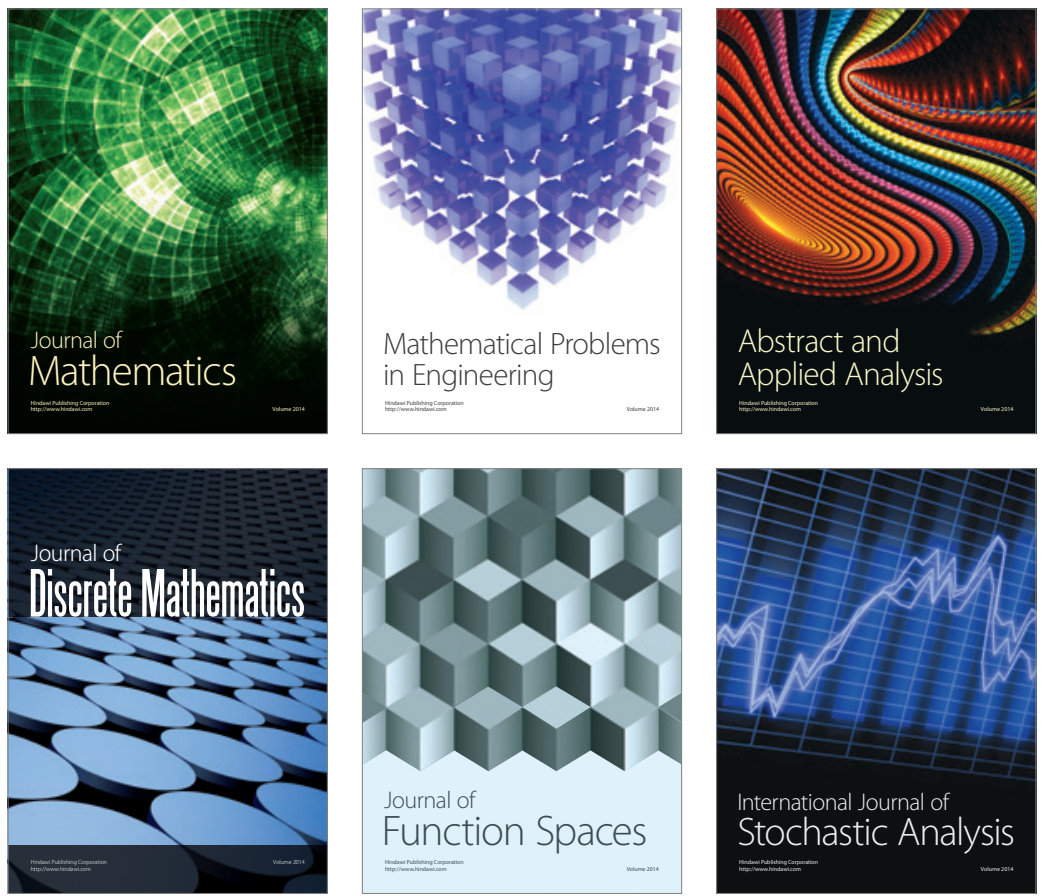

Journal of

Function Spaces

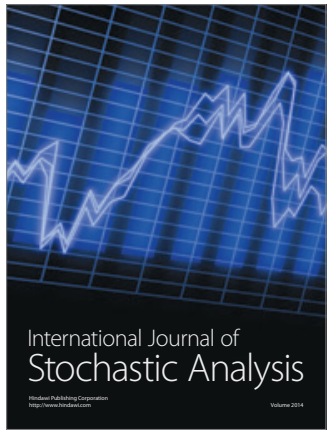

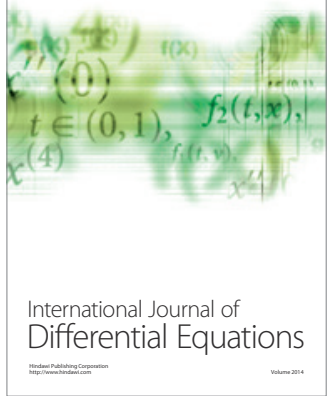
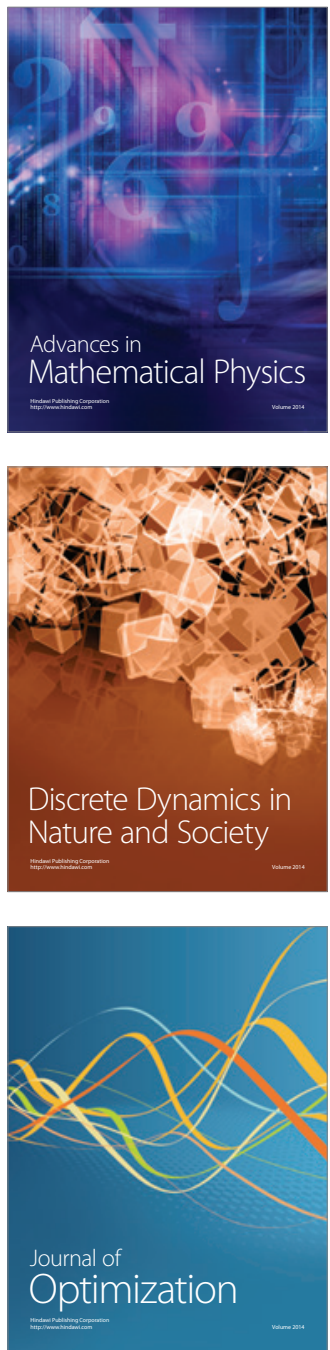